\title{
Validación en México de la Escala de futoverbalizaciones al Hablar en Público
}

Validation of Self-Statements during Public Speaking scale in Mexico

\author{
José Moral de la Rubia \\ César Jesús Antona Casas \\ Cirilo Humberto García Cadena
}

\begin{abstract}
Resumen
La escala de Autoverbalizaciones al Hablar en Público (SSPS; Hofmann \& DiBartolo, 2000), es un instrumento para evaluar cogniciones en la situación de hablar en público, la cual comúnmente genera algún grado de ansiedad. La escala presentó buenas propiedades psicométricas en su estudio original con muestras estadounidenses al igual que en estudios de validación con muestras españolas. A pesar de su importancia, no estaba adaptada en población mexicana, así los objetivos de este estudio fueron: traducir la escala SSPS al español de uso en México, validar su estructura de dos factores correlacionados, calcular su consistencia interna, describir su distribución, contrastar diferencias entre sexos y observar la relación de la SSPS con deseabilidad social, que es un constructo potencialmente vinculado a la ansiedad social. La escala SSPS y el Inventario Balanceado de Deseabilidad Social al Responder (BIDR; Paulhus, 1998), fueron aplicados a una muestra probabilística estratificada por sexos de estudiantes universitarios. La estructura de dos factores correlacionados de autoverbalizaciones negativas y positivas mostró un ajuste adecuado a los datos e invarianza entre ambos sexos por mínimos cuadrados generalizados. La consistencia interna fue alta. La distribución fue asimétrica positiva. La correlación con deseabilidad social fue significativa sobre todo con el factor de autoengaño. Los varones obtuvieron medias más elevadas, pero hubo equivalencia estadística entre las medias de ambos sexos al controlar la deseabilidad social. Se sugiere el uso de la escala SSPS en México, controlando el sesgo de la deseabilidad social.
\end{abstract}

\section{Abstract}

The Self-Statements during Public Speaking (SSPS; Hofmann \& DiBartolo, 2000) scale is an instrument that assesses cognitions in a public speaking situation, which commonly generates some degree of anxiety. The SSPS scale showed good psychometric properties in its original study with American samples in the same way as in its validation studies with Spanish samples. Despite its relevance, it was not adapted in the Mexican population. So the aims of this study were: to translate the SSPS scale into Mexican Spanish, to validate its structure of two correlated factors, to calculate its internal consistency, to describe its distribution, to contrast gender differences, and to observe the relationship of the SSPS scale with social desirability, a construct potentially linked to social anxiety. The SSPS scale and the Balanced Inventory of Desirable Responding (BIDR; Paulhus, 1998) were administered to a probability sample of university students stratified by gender. The structure of two correlated factors of positive and negative self-statements showed an adequate fit to data and invariance between both sexes by Generalized Least Squares. The Internal consistency was high; distribution was positively skewed. The correlation with social desirability was significant, especially with the self-deception factor. Men averaged higher, but the means of both genders were statistically equivalent when social desirability was controlled. We suggest using the SSPS scale in Mexico, controlling the social desirability bias. 
La fobia social o trastorno de ansiedad social se caracteriza por un elevado temor a la evaluación negativa por parte de los demás (American Psychiatric Association, 2000). Como su propio nombre indica, el objeto que causa el temor es de índole social, es decir, son las propias personas quienes se convierten en los estímulos que disparan y exacerban la ansiedad. Al igual que ocurre en cualquier otra fobia o trastorno de ansiedad, la intensidad de los estímulos fóbicos provocará fluctuaciones en la ansiedad. En el caso particular de la fobia social, la relación con los estímulos es aún más interesante, ya que éstos son personas (generalmente fuera del ámbito familiar o del ámbito de las amistades cercanas), que al ser percibidos de modo crítico o potencialmente amenazante pueden suscitar una gran ansiedad y malestar (Antony \& Rowa, 2008; Turk, Heimberg, Luterek, Mennin, \& Fresco, 2005).

La evaluación de la ansiedad y fobia sociales se ha enfocado desde distintas perspectivas, empleándose escalas de autorreporte, escalas de observación y entrevistas (Antona, 2009; García, Olivares \& Vera, 2003). Entre las escalas se puede destacar la de Autoverbalizaciones al Hablar en Público (SSPS) de Hofmann y DiBartolo (2000). Este instrumento evalúa el papel de cogniciones relevantes en una situación específica y generalmente generadora de ansiedad social, como es hablar en público (Heinrichs \& Hofmann, 2001; Hofmann, 2007).

Partiendo de un modelo cognitivo, se plantea que las personas padecen ansiedad social en situaciones de interacción o ejecución porque perciben los estándares sociales (metas y expectativas) de una forma exageradamente elevada. Esto conduce a un incremento en la autoobservación y autoevaluación que impide y entorpece la acción. Las conductas menos habilidosas mantienen o incrementan la ansiedad social hasta alcanzar niveles desadaptativos que interfieren con las rutinas diarias y al final motivan la consulta clínica (Antona \& García, 2008; Hofmann, 2007).

La escala SSPS fue desarrollada basándose en el Social Interaction and Self-Statement Test de Glass, Merluzzi, Biever y Larsen (1982). Este test conductual permite evaluar frecuencia de cogniciones durante una interacción social.

En un primer estudio, con una muestra de 100 estudiantes de la Universidad de Nueva York, la mitad hombres y la mitad mujeres, Hofmann y DiBartolo (2000), seleccionaron los ítems de la SSPS, partiendo de un total de 30. Hallaron una consistencia interna alta $(\alpha=.94)$. En el análisis factorial se identificaron cinco factores. Los dos primeros explicaban el $51 \%$ de la varianza total. Así se minimizó el número de ítems considerando sólo los que tenían su saturación más alta en uno de los dos primeros factores (> .60). El primero se definió como factor de autoverbalizaciones positivas (SSPS-P) con 5 ítems y el segundo como factor de autoverbalizaciones negativas (SSPS-N) también con 5 ítems. El alfa de Cronbach fue alta tanto para SSPS-P $(\alpha=.84)$ como para SSPS-N $(\alpha=$ .83). El promedio del puntaje total de la muestra de mujeres fue significativamente menor que el de hombres; el factor de autoverbalizaciones negativas era el que generaba la diferencia.

En un segundo estudio, con una muestra de 201 estudiantes femeninas del Smith College en Northampton, Massachusetts, Hofmann y DiBartolo (2000), reprodujeron los dos factores con un alfa de Cronbach de .75 para SSPS-P y de .86 para SSPS-N. También estudiaron la validez convergente con depresión. La correlación entre el Inventario de Depresión de Beck (Beck Depression Inventory, BDI; Beck, Ward, Mendelson, Mock \& Erbaugh, 1961) y SSPS fue significativa, directa y baja $(r=.21)$.

En un tercer estudio, con una muestra de 41 pacientes diagnosticados de fobia social, Hofmann y DiBartolo (2000) encontraron tanto coeficientes de alfa de Cronbach altos para SSPS- 
$\mathrm{P}(\alpha=.80)$ y SSPS-N $(\alpha=.86)$ como correlaciones test-retest elevadas, con un intervalo de 3 meses, para SSPS-P $(r=.78)$ y para SSPS-N $(r=.80)$. Se reprodujo la estructura de dos factores correlacionados. El promedio del puntaje total difirió significativamente de los obtenidos en las muestras de estudiantes de los dos estudios anteriores, manifestando una mayor frecuencia de autoverbalizaciones negativas y menor frecuencia de autoverbalizaciones positivas. En las dos muestras de estudiantes dominaron las autoverbalizaciones positivas sobre las negativas, pero en la clínica los promedios fueron equivalentes. Además, la subescala de autoverbalizaciones negativas fue sensible a los cambios producidos por intervención terapéutica conducida entre estos pacientes, pero no la de autoverbalizaciones positivas.

En un cuarto estudio con 19 participantes elegidas aleatoriamente de los tercios de puntuaciones superiores y superiores en la subescala SSPS-N de una muestra de 100 estudiantes femeninas, Hofmann y DiBartolo (2000) hallaron que el factor de autoverbalizaciones negativas fue más sensible para diferenciar ambos grupos en un experimento de hablar en público, lo cual validó el resultado de la intervención clínica, pero en población de estudiantes.

De estos 4 estudios Hofmann y DiBartolo (2000) concluyeron que la escala SSPS es un instrumento breve con gran potencial para su uso tanto en población clínica como abierta por sus propiedades de consistencia, estabilidad, sensibilidad al cambio y validez.

Rivero, García y Hofmann (2010), en una muestra comunitaria de 1,694 adolescentes españoles, reprodujeron la estructura de dos factores correlacionados con buenos índices de ajuste $(S R M R=.04, G F I=.99, A G F I=.98$, $N F I=.98, N N F I=.98$ y $C F I=0.98)$, siendo la consistencia interna de $\alpha=.86$ para SSPS-N y .69 para SSPS-P. Por otra parte, Gallego, Botella, García, Quero y Baños (2010), validaron la escala en población española con una muestra clínica de 121 participantes con un diagnóstico de fobia social. El análisis factorial exploratorio indicó la existencia de dos factores: autoverbalizaciones positivas (SSPS-P) y autoverbalizaciones negativas (SSPS-N) conforme a las expectativas. La consistencia interna fue alta para ambos factores: $\alpha=.81$ para SSPS-P y $\alpha=.88$ para SSPS-N; además la escala SSPS discriminó bien y de forma consonante con la literatura entre distintos tipos de fobia social.

En general, las distribuciones de las escalas que evalúan ansiedad y fobia social son asimétricas positivas o con sesgo hacia los valores por debajo de la media, al igual que sus dos factores. Las medias de los hombres tienden a ser mayores que las de las mujeres y presentan correlación negativa con la edad, es decir, a más edad menos fobia (Antony \& Rowa, 2008).

El empleo de escalas de deseabilidad social en los estudios sobre fobia social no es usual, pero su inclusión tiene bastante sentido. Las personas con fobia social tratan de crear una impresión positiva en los demás. El temor principal de la fobia social radica en el miedo a la evaluación negativa por parte de los demás, por lo cual es esperable que quienes lo sufran presenten deseabilidad social (Antony, Rowa, Liss, Swallow, \& Swinson, 2005).

Para evaluar este aspecto es especialmente útil el modelo bifactorial de manejo de la impresión o fingimiento y autoengaño o falta de crítica hacia sí mismo de Paulhus (2002), el cual se halla instrumentalizado por el Inventario Balanceado de Deseabilidad Social al Responder (BIDR-6). En relación con la fobia social, cuya fenomenología implica vergüenza e inferioridad en la evaluación interna, es probable que correlacione más con autoengaño que con manejo de la impresión. La persona bajo un sesgo egotista no reconoce todos sus aspectos fóbicos sociales e interiormente se define como más segura y hábil en la interacción social de lo que realmente es. Esto puede ser especialmente relevante en la situación de hablar en público entre estudiantes universitarios, al ser 
importante en el desempeño de su rol como estudiante y profesional.

De lo anteriormente expuesto se colige que la escala SSPS es un instrumento que evalúa aspectos cognitivos relevantes en ansiedad social, presenta buenas propiedades psicométricas y ha sido validado con éxito en España.

La presencia de fobia social en estudiantes universitarios abocados a profesiones de servicio, como aquellas de área socio-sanitario o docencia, no solamente genera un sufrimiento y malestar internos, sino también constituye un obstáculo importante para el desarrollo profesional. En estos casos, el temor y la evitación de situaciones sociales se reflejarán primero en el rendimiento en materias de práctica o que requieran trabajo en equipo; posteriormente, afectará el ejercicio profesional, e incluso generar la postergación de su inicio o la deserción una vez iniciado (Stewart \& Mandrusiak, 2007). Durante el periodo estudiantil, el hablar en público al realizar trabajos en equipo, exponer trabajos o dar exámenes orales es una situación especialmente sensible (Antona, Delgado, García, \& Estrada, 2012). De aquí surge la importancia de la evaluación y abordaje terapéutico de la fobia social específica en la situación de hablar en público en los estudiantes universitarios de carreras como psicología y orientación deportiva.

Considerando que la escala SSPS es un instrumento de evaluación de fobia social específica, comúnmente empleado en diversas investigaciones publicadas en revistas internacionales y de impacto, posee buenas propiedades psicométricas para evaluar ansiedad social en la situación de hablar en público y no está validado en México, pero sí en otro país hispanoparlante, este estudio tiene como objetivos: 1) traducir la escala SSPS al español de uso en México, 2) validar su estructura de dos factores correlacionados y contrastar su invarianza entre ambos sexos, 3) calcular la consistencia interna del puntaje total y sus dos factores, 4) describir las distribuciones de los mismos, 5) determinar diferencias de tendencia central entre los se- xos, y 6) observar la relación de la escala SSPS con deseabilidad social. El estudio se realizó en una muestra de estudiantes universitarios por la relevancia de evaluar la fobia social específica en la situación de hablar en público dentro de esta población y en consonancia con el estudio original de esta escala.

Se esperan dos factores correlacionados, uno de autoverbalizaciones positivas y otro de autoverbalizaciones negativas, invarianza de este modelo factorial entre mujeres y hombres, consistencia interna alta, siendo ligeramente más consistente el de autoverbalizaciones negativas, mayor promedio en autoverbalizaciones positivas que negativas, distribuciones asimétricas en el puntaje total y los factores, mayor promedio en hombres y correlación baja con deseabilidad social más con el factor de autoengaño que con el de manejo de la impresión.

\section{Método}

\section{Participantes}

La población objeto de estudio fueron estudiantes universitarios, como también se empleó en el estudio original. Se realizó un muestreo probabilístico por estratos equivalentes de sexos. Se trabajó con dos facultades de la Universidad Autónoma de Nuevo León (UANL): Psicología (con mayoría de mujeres) y Organización Deportiva (con mayoría de hombres). Entre ambas suman un total de 2,500 alumnos de licenciatura con equivalencia de sexos. Se tomó como parámetro la media del puntaje total de la escala SSPS para estimar el tamaño de la muestra. Al ser la desviación estándar de la distribución del SSPS en la presente muestra de 5.71 , con un tamaño de muestra de 624 participantes y un nivel de confianza de $95 \%$, el error absoluto fue de 0.38 . Este cálculo se realizó con el programa Win Episcope de Thrusfield, Ortega, De Blas, Noordhuizen y Frankena (2001).

La muestra de 624 participantes quedó integrada por 312 hombres y 312 mujeres. La media de edad fue de 19.15 años $(D E=2.13)$. El 
52\% (324 de 624) de la muestra procedió de la Facultad de Psicología y 48\% (300 de 624) de la Facultad de Orientación Educativa, siendo los porcentajes estadísticamente equivalentes (prueba binomial: $p=.36$ ). Las distribuciones de los sexos fueron diferenciales entre ambas facultades $(\chi 2[1, N=624]=97.44, p<.01$; con la corrección de Yates: $\chi 2[1, N=624]=90.91$, $p<.01$ ). En Psicología, el 70\% (210 de 300) de los participantes fueron mujeres y 30\% (90 de 300) hombres. En la Facultad de Orientación Educativa, el 68.5\% (222 de 324) de los participantes fueron hombres y $31.5 \%$ (102 de 324) mujeres. La media de edad fue equivalente entre ambas facultades $(t[622]=1.56, p=.12)$ y entre ambos sexos $(t[599.15]=-0.84, p=.40)$.

\section{Instrumentos}

La Escala de Autoverbalizaciones al Hablar en Público (SSPS; Hofmann \& DiBartolo, 2000), consta de 10 ítems con un formato de respuesta de seis puntos que miden las autoverbalizaciones asociadas con la situación de hablar en público. Se compone de dos factores: autoverbalizaciones positivas (SPS-P) con 5 ítems (1, $3,5,6$ y 9) y autoverbalizaciones negativas (SSPS-N) también con 5 ítems (2, 4, 7, 8 y 10). Véase la escala traducida en el anexo, en la que se manejó un rango de cinco puntos por ítem por las sugerencias recibidas en el piloteo de compresión.

El Inventario Balanceado de Deseabilidad Social al Responder (BIDR-6; Paulhus, 1998), consta de 40 ítems redactados como proposiciones afirmativas, la mitad en sentido de respuestas socialmente deseables (directos) y la otra mitad en sentido opuesto (inversos). Se responden según una escala tipo Likert que va del 1 (no cierto) hasta el 7 (muy cierto). Para su calificación, primero, se invierten los 20 ítems con redacción opuesta al rasgo $(2,4,6,8,10$, $12,14,16,18,20,21,23,25,27,29,31,33,35$, 37 y 39 ), restando su puntuación a 8 . A continuación el puntaje total se obtiene sumando los 40 ítems. Consta de dos factores: auto-engaño (ítems del 1 al 20) y manejo de impresión (ítems del 21 al 40). Cada factor cuenta con la mitad de sus ítems en sentido inverso. Paulhus (1998), con diversas muestras de población general, observó que la consistencia interna varió de .68 a .80 para el factor de auto-engaño, y de $.75 \mathrm{a}$ .86 para el factor de manejo de impresión; mientras que al juntar los 40 ítems resultó de .83.

\section{Procedimiento}

Las dos escalas (SSPS y BIDR) fueron traducidas por el procedimiento de traducción reversa (García, 2009). Primero se tradujeron al español por un lingüista experto, luego se volvieron a traducir al inglés por un segundo lingüista experto. Se evaluó la equivalencia semántica de ambas traducciones por otro tercer lingüista en una escala de 5 "totalmente equivalente" a 1 "totalmente disimilares". Todos aquellos ítems con menos de 4 puntos se tradujeron de nuevo por un psicólogo experto y se evaluó su equivalencia semántica con el original por el tercer lingüista experto. Esto afectó a un ítem de SSPS y tres de BIDR. En la segunda traducción al español los 4 ítems alcanzaron valores de equivalencia semántica altos (4 o 5). A continuación se evaluó la comprensibilidad de las escalas en una muestra de 30 participantes con estudios de primaria. La escala de evaluación fue de tres puntos: $3=$ comprensible, 2 = genera dudas, 1 = no lo comprendo. Se exigió para cada ítem que al menos 25 de los 30 participantes $(80 \%)$ señalasen comprenderlo (puntuación 3). En caso de marcar 1 ó 2 se pidió indicar por qué. A partir de los señalamientos se hicieron modificaciones.

Estas dos escalas fueron administradas junto a otras tres de fobia social en un cuestionario que se tardaba en responder de 20 a 40 minutos. Para evitar el efecto de la fatiga y orden fijo de secuencia en los resultados de cada escala se empleó el método de la equiponderación total: 24 series cada una, aplicada 26 veces, la mitad en la Facultad de Psicología y la otra mitad en la Facultad de Organización Educativa. 
La aplicación fue individual para garantizar la independencia de los participantes, los cuales fueron seleccionados de forma aleatoria. El muestreo se realizó de junio a diciembre de 2010. La participación en la encuesta fue voluntaria y anónima. Se requirió conceder el consentimiento expreso en la primera página del cuestionario para participar tras garantizarse la confidencialidad de la información proporcionada. En caso de que algún participante solicitase ayuda psicológica, éste fue derivado a la unidad clínica de la facultad, respetándose de este modo las recomendaciones éticas de la American Psychological Association (2002), así como las de la Sociedad Mexicana de Psicología (2007).

\section{Análisis de datos}

La consistencia interna se estimó por el coeficiente alfa de Cronbach $(\alpha)$. Se consideraron valores de consistencia interna altos los mayores o iguales a .70, adecuados los mayores o iguales a .60 y bajos los menores de .60 (Cronbach \& Shavelson, 2004). El ajuste de la distribución a una curva normal se contrastó por la prueba de Kolmogorov-Smirnov $\left(Z_{K-S}\right)$. Las diferencias de medias entre factores se contrastaron por la $t$ de Student para datos emparejados, y entre hombres y mujeres por la $t$ de Student para muestras independientes. La correlación entre SSPS y BIDR se calculó por el coeficiente producto-momento de Pearson. Estos cálculos se realizaron con el programa SPSS12 (SPSS Incorporation, 2007).

La estructura dimensional se determinó tanto por análisis factorial exploratorio (AFE) por Componentes Principales con rotación Oblimín como por análisis factorial confirmatorio (AFC) por Mínimos Cuadrados Generalizados. El cumplimiento del supuesto de normalidad multivariada se valoró desde la curtosis multivariada de Mardia y su razón crítica; valores menores que 10 y 3 , respectivamente, reflejan buen cumplimiento. Se contemplaron siete índices de ajuste: tres básicos (función de discrepancia
[FD], prueba chi-cuadrada [ $\chi 2]$ y cociente entre chi-cuadrada y sus grados de libertad $[\chi 2 / g /])$; dos poblacionales de no centralidad (parámetro de no centralidad poblacional [PNCP] y error cuadrático medio de aproximación [RMSEA] de Steiger-Lind); y dos índices comparativos (índice de bondad de ajuste [GFl] de Jöreskog y Sörbom y su modalidad corregida [AGFI]). Se estipularon como valores de buen ajuste para los índices: $p$ de $\chi 2 \geq .05, \chi 2 / g l \leq 2, F D$ y $P N C P$ $\leq$ un cuarto de los valores correspondientes al modelo independiente, RMSEA $\leq .05, \mathrm{GFI} \geq .95$ y $A G F I \geq .90$; y como valores adecuados: $p$ de $\chi 2 \geq .01, \chi 2 / g l \leq 3, F D$ y $P N C P \leq$ tres cuartos de los valores correspondientes al modelo independiente, RMSEA $\leq .08, \mathrm{GFI} \geq .85$ y $A G F I \geq$ .80. La parsimonia del modelo se estimó desde la razón de parsimonia $(R P)$ de James-MulaikBrett (alta $\geq .66$, media $\geq .33$ y baja $<.33$ ). La adecuación del tamaño muestral se juzgó desde el tamaño crítico de Hoelter; se consideró muy adecuado si el tamaño crítico era mayor que el muestral con un nivel de significación de .01 y adecuado con nivel de significación de .05 (Kline, 2010; Moral, 2006). Se realizó un contraste multigrupo del modelo factorial para probar su invarianza entre mujeres y hombres; en este contraste se estimaron cuatro modelos anidados por constricción de parámetros: sin constricciones, con constricciones en los pesos de medida, en las varianza-covarianzas y en los residuos de medida. Para considerar el modelo invariante entre ambos sexos, se requirió parámetros significativos en las dos muestras en los 4 modelos, ajuste bueno o adecuado y equivalencia estadística de la bondad de ajuste entre los cuatro modelos. Se comparó la bondad de ajuste diferencial entre modelos anidados por la prueba de la diferencia de los estadísticos chi-cuadrado $(\Delta \chi 2)$, considerando un nivel de significación más estricto $(\alpha=.05)$ o menos estricto ( $\alpha=.01$ ) (Byrne, 2008). Los cálculos del análisis factorial exploratorio se realizaron con el SPSS16 y los del confirmatorio con AMOS 16 (Arbuckle, 2007). 
La potencia de los contrastes por análisis factorial confirmatorio se estimó desde el error de aproximación cuadrático medio (RMSEA). La estimación se hizo con un nivel de significancia de .05. Se empleó como hipótesis nula el valor medio de la RMSEA del modelo independiente (mínima constricción) y como hipótesis alternativa el valor medio de la RMSEA del modelo contrastado (mayor constricción) (MacCallum, Browne, \& Cai, 2006). El cálculo de la potencia se hizo con el programa de Preacher y Coffman (2006).

Con el objetivo de conocer el efecto del sexo y debido a la composición diferencial de mujeres y hombres entre las dos facultades, el contraste multigrupo del modelo factorial se realizó también por facultades. Por otra parte, la comparación de medias en la puntuación total y los factores de SSPS se realizó entre las dos facultades por la $t$ de Student para muestras independientes; asimismo, se parcializó el efecto de la facultad sobre la puntuación total y los factores del SSPS en la comparación entre ambos sexos por medio del análisis de covarianza.

\section{Resultados}

\section{Estructura factorial}

Con base en el criterio de Kaiser (autovalores de la matriz de correlaciones mayores a 1) se definieron dos componentes que explicaron el $44.36 \%$ de la varianza total. Al aplicar la rotación Oblimín se definió un primer componente con los cinco ítems directos de autoverbalizaciones negativas (ítems 2, 4, 7, 8 y 10) y un segundo componente con los cinco ítems inversos de autoverbalizaciones positivas (ítems 1, 3, 5, 6 y 9). Todas las cargas factoriales fueron mayores a .50 y la consistencia interna no se incrementó al eliminar ningún ítem. La correlación entre ambos fue significativa, baja y negativa $(r=-.24, p<.01)$ (véase tabla 1$)$.

Tabla 1.

Matriz de patrones y estructural

\begin{tabular}{lllll}
\hline \multirow{2}{*}{ Ítems } & \multicolumn{2}{c}{ Patrones } & \multicolumn{2}{c}{ Estructural } \\
\cline { 2 - 5 } & C1 & C2 & C1 & C2 \\
\hline $\begin{array}{l}\text { 7. Lo que yo diga probablemente sonará } \\
\text { estúpido }\end{array}$ & .78 & .01 & .78 & -.17 \\
$\begin{array}{l}\text { 8. Probablemente "la vaya a regar" de todas } \\
\text { maneras }\end{array}$ & .75 & .09 & .73 & -.09 \\
$\begin{array}{l}\text { 10. Me siento incómodo y tonto, se van a dar } \\
\text { cuenta de cómo me siento }\end{array}$ & .71 & .06 & .69 & -.11 \\
$\begin{array}{l}\text { 4. Fracasar en esta situación sería una } \\
\text { prueba más de mi incapacidad }\end{array}$ & .56 & -.03 & .57 & -.16 \\
$\begin{array}{l}\text { 2. Soy un perdedor } \\
\begin{array}{l}\text { 5. Aun cuando las cosas no salgan bien, no } \\
\text { es una catástrofe }\end{array}\end{array}$ & .52 & -.20 & .56 & -.32 \\
$\begin{array}{l}\text { 9. En lugar de preocuparme, podría } \\
\text { concentrarme en lo que quiero decir }\end{array}$ & .13 & .72 & -.05 & .69 \\
$\begin{array}{l}\text { 3. Esta es una situación incómoda, pero } \\
\text { puedo manejarla }\end{array}$ & -.03 & .64 & -.18 & .64 \\
$\begin{array}{l}\text { 1. No tengo nada que perder, vale la pena el } \\
\text { intentarlo }\end{array}$ & -.12 & .57 & -.26 & .60 \\
\begin{tabular}{l} 
6. Puedo manejar cualquier cosa \\
\hline
\end{tabular} & -.24 & .52 & -.36 & .58 \\
\hline
\end{tabular}

Extracción: Componentes Principales. Rotación: Oblimín: La solución convergió en 5 iteraciones. C1: Autoverbalizaciones negativas y C2: Autoverbalizaciones positivas. 
Se contrastó la estructura de dos factores correlacionados por análisis factorial confirmatorio. La curtosis multivariada de Mardia de los 10 ítems fue 29.99 y su razón crítica 17.10 , lo que refleja incumplimiento del supuesto de normalidad multivariada. La parsimonia del modelo fue alta $(R P=.76)$. El modelo presentó todos sus parámetros significativos. De los 7 índices de ajuste, 5 fueron buenos ( $G F I=.97, A G F I=$ $.96, F D=0.13, P N C P=0.08$ y $R M S E A=.05)$, uno adecuado $(\chi 2 / g l=2.40)$ y uno malo $(\chi 2[34$, $N=624]=81.61, p<.01)$. Desde el tamaño crítico de Hoelter el tamaño muestral fue muy adecuado y la potencia del contraste perfecta (véanse figura 1 y tabla 2).

Modelo de dos factores correlacionados

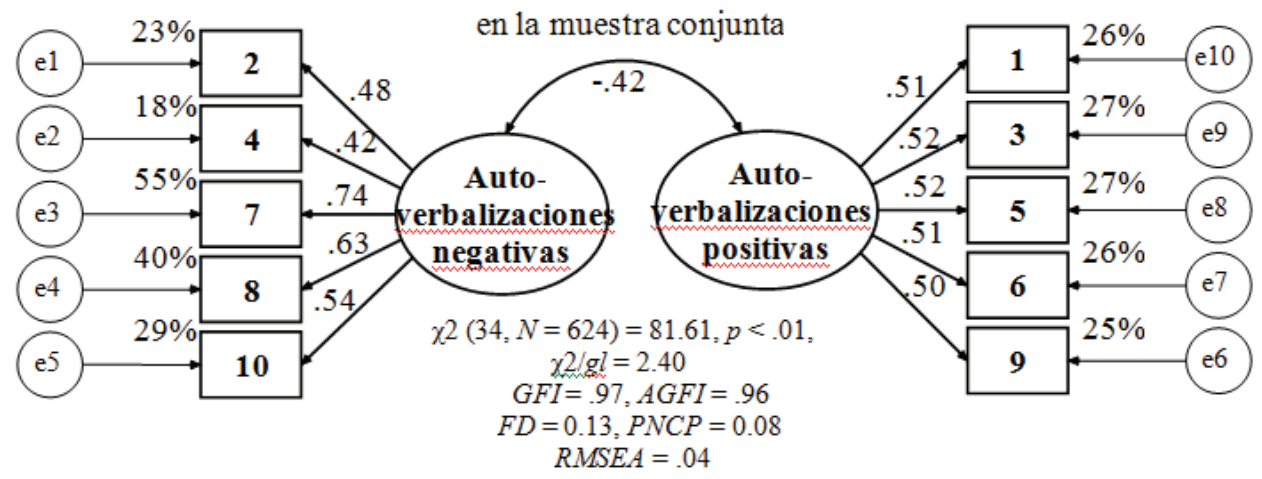

Figura 1. Modelo estandarizado estimado por Mínimos Cuadrados Generalizados (GLS).

Tabla 2.

Índices de ajuste, adecuación del tamaño muestral y potencia del contraste

\begin{tabular}{|c|c|c|c|c|c|c|c|}
\hline \multirow{2}{*}{ Estadísticos } & \multicolumn{2}{|c|}{ Interpretación } & \multirow{2}{*}{ Unigrupo } & \multicolumn{4}{|c|}{ Multigrupo sexos. Constricciones: } \\
\hline & Bueno & malo & & $\operatorname{Sin}$ & Pes. & Cov. & Res. \\
\hline$x^{2}$ & & & 81.61 & 122.43 & 130.73 & 131.93 & 194.89 \\
\hline$g l$ & & & 34 & 68 & 76 & 79 & 89 \\
\hline$p$ & $>.05$ & $<.01$ & $<.01$ & $<.01$ & $<.01$ & $<.01$ & $<.01$ \\
\hline$\chi 2 / g l$ & $\leq 2$ & $>3$ & 2.40 & 1.80 & 1.72 & 1.67 & 2.19 \\
\hline GFI & $\geq .95$ & $<.85$ & .97 & .96 & .96 & .96 & .94 \\
\hline$A G F I$ & $\geq .90$ & $<.80$ & .96 & .94 & .94 & .94 & .92 \\
\hline$F D$ & $<1 / 4 F D_{1}$ & $>3 / 4 F D_{1}$ & 0.13 & 0.20 & 0.21 & 0.21 & 0.31 \\
\hline$P N C P$ & $<1 / 4 P N C P_{1}$ & $>3 / 4 P N C P_{1}$ & 0.08 & 0.09 & 0.09 & 0.09 & 0.17 \\
\hline RMSEA & $\leq .05$ & $>.09$ & .05 & .04 & .03 & .03 & .04 \\
\hline$\alpha=.05$ & & $>N$ & 372 & 450 & 465 & 477 & 350 \\
\hline$\alpha=.01$ & $\leq N$ & & 428 & 500 & 513 & 526 & 394 \\
\hline$\phi$ & $\geq .90$ & $<.80$ & 1 & .99 & 1 & 1 & .99 \\
\hline
\end{tabular}

Constricciones anidadas: sin constricciones ( $\sin$ ), en los pesos de medida (pes.), varianza-covarianzas (cov.) y residuos de medida (res.). Índices de ajuste: $\chi 2$ = prueba de bondad de ajuste chi-cuadrado, $g l=$ grados de libertad del estadístico chi-cuadrado, $p=$ probabilidad de mantener la hipótesis nula de bondad de ajuste del estadístico chi-cuadrado, $\chi 2 / g l=$ cociente entre el estadístico chi-cuadrado y sus grados de libertad, $F D$ = valor de la función de discrepancia, $G F I=$ índice de bondad de ajuste de Jöreskog y Sörbom, $A G F I=$ índice de bondad de ajuste ajustado de Jöreskog y Sörbom, $P N C P=$ valor medio del parámetro de no centralidad poblacional, y RMSEA = valor medio de error cuadrático medio de aproximación de Steiger-Lind.

$N_{\text {critica }}=$ tamaño de muestra crítico de Hoelter con un nivel de significación de .05 o .01 para mantener la hipótesis nula de bondad de ajuste con el estadístico chi-cuadrado y grados de libertad del modelo contrastado. $N=624$.

$\phi=$ potencia para un nivel de significación de .05 , teniendo como hipótesis nula la RMSEA del modelo independiente (.10 en el contraste unigrupo y .07 en el multigrupo) y como alternativa la del modelo contrastado.

El valor de la función de discrepancia del modelo independiente $(F D I)$ fue 0.62 en el contraste unigrupo y 0.54 en el multigrupo. El valor medio del parámetro de no centralidad poblacional del modelo independiente $(P N C P I)$ fue 0.47 en el contraste unigrupo y multigrupo. 
Al realizar el contraste multigrupo del modelo entre hombres y mujeres, todos los parámetros fueron significativos en ambas muestras, ya sea en el modelo sin constricciones o en los tres modelos con constricciones anidadas. De los 7 índices de ajuste, 5 fueron buenos ( $\chi 2$ / $g l=1.80, R M S E A=.04, P N C P=0.09, G F I=$ .96 y $A G F I=.94)$, uno adecuado $(F D=0.20)$ y uno malo $(\chi 2[68, N=624]=122.43, p<.01)$. Por la prueba de la diferencia de los estadísticos chi-cuadrado entre pares de modelos anidados, con un nivel de significación de .05, la bondad de ajuste del modelo sin constricciones fue equivalente al modelo con constricciones en los pesos de medida $(\chi 2[8, N=624]=8.29$, $p=.41)$ y con constricción adicional en las varianza-covarianzas $(\chi 2[11, N=624]=9.49, p=$ $.58)$; igualmente, la bondad de ajuste del modelo con constricción en los pesos de medida fue equivalente al modelo con constricción adicional en las varianza-covarianzas $(\chi 2[3, N=624]$ $=1.20, p=.75)$. El modelo con constricción adicional en los residuos de medida tuvo diferencialmente peor ajuste en comparación con los tres anteriores con menos constricciones $(p<$ .01). Desde el tamaño crítico de Hoelter el tamaño muestral fue muy adecuado en los cuatro modelos anidados y la potencia del contraste perfecta o casi perfecta (véase tabla 2)

Debido a la integración desigual de los sexos en las muestras de ambas facultades, se contrastó la invarianza factorial entre los estudiantes de ambas facultades. Todos los parámetros fueron significativos, ya sea en el modelo sin constricciones o en los tres modelos con constricciones anidadas. De los 7 índices de ajuste, 5 fueron buenos $(\chi 2 / g l=1.84, R M S E A=.04$, $P N C P=0.09, G F I=.96$ y $A G F I=.94)$, uno adecuado $(F D=0.20)$ y uno malo $(\chi 2[68, N=$ $624]=124.81, p<.01)$. Por la prueba de la diferencia de los estadísticos chi-cuadrado, con un nivel de significación de .01, la bondad de ajuste del modelo sin constricciones fue equivalente al modelo con constricción en los pesos de medida $(\chi 2[8, N=624]=18.66, p=.02)$ y con constricción adicional en las varianza-covarianzas $(\chi 2[11, N=624]=22.93, p=.02) ;$ asimismo, la bondad de ajuste del modelo con constricción en los pesos de medida fue equivalente al modelo con constricción adicional en las varianza-covarianzas con un nivel de significación de $.05(\chi 2[3, N=624]=4.26, p=.23)$. El modelo con constricción adicional en los residuos de medida tuvo diferencialmente peor ajuste en comparación con los tres anteriores $(p<.01)$. El tamaño muestral fue muy adecuado en los cuatro modelos anidados según el tamaño crítico de Hoelter y la potencia del contraste perfecta o casi perfecta.

\section{Consistencia interna y distribución}

En la muestra conjunta la consistencia interna de los 10 ítems (todos puntuados en sentido de verbalizaciones que manifiestan ansiedad social) fue alta $(\alpha=.70)$ y su distribución fue asimétrica positiva $(S=0.49, E E=0.10)$, no ajustándose a una curva normal. El factor de autoverbalizaciones negativas presentó una consistencia interna adecuada $(\alpha=.69)$ y su distribución también fue asimétrica positiva ( $S=0.86, E E=0.10)$, no ajustándose a una curva normal. El factor de autoverbalizaciones positivas (invertidas las puntuaciones) también mostró una consistencia interna adecuada ( $\alpha$ $=.64)$ y su distribución fue asimétrica positiva $(S=0.84, E E=0.10)$ y apuntada $(C=.81, E E$ $=.20$ ), tampoco ajustándose a una curva normal (véase tabla 3). La media de 10.53 ( $D E=$ 3.65) en autoverbalizaciones positivas (con las puntuaciones invertidas) fue significativamente mayor $(t[623]=9.20, p<.01$, siendo $r=.26$, $p<.01)$ que la media de $8.92(D E=3.54)$ en autoverbalizaciones negativas. 
Tabla 3.

Consistencia interna, distribución y ajuste a la normalidad

\begin{tabular}{|c|c|c|c|c|}
\hline \multicolumn{2}{|c|}{ Estadísticos } & \multirow{2}{*}{$\begin{array}{c}\text { Puntaje total } \\
10\end{array}$} & \multirow{2}{*}{$\frac{\text { SSPS-N }}{5}$} & \multirow{2}{*}{$\begin{array}{c}\text { SSPS-P } \\
5\end{array}$} \\
\hline Consistencia & No. Ítems & & & \\
\hline interna & $\alpha$ & .70 & .69 & .64 \\
\hline & Rango & $10-50$ & $5-25$ & $5-25$ \\
\hline & $M$ & 19.44 & 8.92 & 10.53 \\
\hline & $D E$ & 5.71 & 3.54 & 3.65 \\
\hline & $S$ & 0.49 & 0.86 & 0.84 \\
\hline & $C$ & -0.33 & 0.30 & 0.81 \\
\hline & P10 & 12 & 5 & 6 \\
\hline \multirow{8}{*}{$\begin{array}{l}\text { Descriptivos } \\
\text { de la } \\
\text { distribución }\end{array}$} & $P 20$ & 14 & 5 & 7 \\
\hline & P30 & 16 & 6 & 9 \\
\hline & P40 & 17 & 7 & 9 \\
\hline & P50 & 19 & 8 & 10 \\
\hline & P60 & 21 & 9 & 11 \\
\hline & P70 & 22 & 10 & 12 \\
\hline & P80 & 24 & 12 & 13 \\
\hline & $P 90$ & 28 & 14 & 16 \\
\hline \multirow{2}{*}{$\begin{array}{l}\text { Ajuste a la } \\
\text { normalidad }\end{array}$} & $Z_{K-S}$ & 2.18 & 3.35 & 3.31 \\
\hline & $p$ & $* *$ & $* *$ & ** \\
\hline
\end{tabular}

$N=624 . E E$ de $S=0.10$ y $E E$ de $C=.20 .{ }^{* *} p<.01$.

Autoverbalizaciones negativas (SSPS-N): ítems 2, 4, 7, 8 y 10, y positivas (SSPS-P): ítems $1,3,5,6$ y 9 .

Los hombres promediaron significativamente más alto en el puntaje total $(t[622]=2.39, p=$ .02) y en el factor de autoverbalizaciones negativas $(t[622]=2.05, p=.04)$. La media del factor de autoverbalizaciones positivas fue estadísticamente equivalente entre ambos sexos $(t[622]$ $=-1.58, p=.11$ ) (véase tabla 4 ).

Tabla 4.

Diferencia de medias entre ambos sexos

\begin{tabular}{cccccccccccc}
\hline \multirow{2}{*}{ SSPS } & \multicolumn{2}{c}{ Hombres } & \multicolumn{2}{c}{ Mujeres } & \multicolumn{2}{c}{ Levene } & \multicolumn{3}{c}{ Student } & \multicolumn{3}{c}{ Diferencia } \\
& $M$ & $D E$ & $M$ & $D E$ & $F$ & $p$ & $T$ & $g l$ & $p$ & $M$ & $E E$ \\
\hline Puntaje total & 19.96 & 5.63 & 18.92 & 5.75 & 0.12 & .73 & 2.29 & 622 & .02 & 1.04 & 0.46 \\
SSPS-N & 9.21 & 3.56 & 8.62 & 3.50 & 0.26 & .61 & 2.05 & 622 & .04 & 0.58 & 0.28 \\
SSPS-P & 10.76 & 3.70 & 10.29 & 3.60 & 0.75 & .39 & 1.58 & 622 & .11 & 0.46 & 0.29 \\
\hline
\end{tabular}

312 hombres y 312 mujeres. Autoverbalizaciones negativas (SSPS-N): ítems 2, 4, 7, 8 y 10, y positivas (SSPSP): ítems 1, 3, 5, 6 y 9. 
Debido a la integración desigual de los sexos en las muestras de ambas facultades, se contrastó la diferencia por facultades. Los promedios fueron estadísticamente equivalentes entre ambas facultades tanto en la puntuación total $(t[622]=-1.27, p=.21)$ como en los factores de autoverbalizaciones negativas $(t[622]=-0.96, p$ $=.34) \mathrm{y}$ autoverbalizaciones positivas $(t[622]=$ $1.05, p=.30)$. Así, si se parcializa el efecto de la facultad, esto no afecta a la significación de las diferencias entre sexos en la puntuación total y factores de SSPS.

\section{Correlación entre SSPS y BIDR}

La consistencia interna de los 4 factores del BIRD varió de .61 a .76. Por el método de Mínimos Cuadrados Generalizados los índices de ajuste de una estructura de 4 factores co- rrelacionados fueron de buenos $(\chi 2 / \mathrm{gl}=1.77 \mathrm{y}$ RMSEA $=.03$ ) a adecuados (FD = 2.09, PNCP $=0.91, \mathrm{GFI}=.90$ y AGFI $=.88)$ y mejoraron al considerar sólo los 10 ítems directos de autoengaño y los 10 directos de manejo de la impresión para definir un modelo de dos factores correlacionados $(F D=0.47, \chi 2 / g l=1.74, P N C P$ $=0.20, \mathrm{RMSEA}=.03, \mathrm{GFI}=.95$ y $\mathrm{AGFI}=.94)$; los valores de consistencia interna de estos dos factores son mayores a .70 .

Las correlaciones entre SSPS y BIDR fueron significativas sobre todo con los factores de ítems directos, destacando el autoengaño sobre el manejo de la impresión, siendo ligeramente más altas en mujeres que en hombres. La correlación de los puntajes totales de ambas escalas fue de -.42 , lo que supone $18 \%$ de varianza compartida (véase tabla 5).

Tabla 5.

Correlaciones entre SSPS y BIDR en la muestra conjunta, de hombres y mujeres

\begin{tabular}{|c|c|c|c|c|c|c|c|c|c|}
\hline \multirow{3}{*}{$\begin{array}{l}\text { Deseabilidad } \\
\text { social }\end{array}$} & \multicolumn{9}{|c|}{ Autoverbalizaciones al Hablar en Público (SSPS) } \\
\hline & \multicolumn{3}{|c|}{ Conjunta $(N=624)$} & \multicolumn{3}{|c|}{ Hombres $(n=312)$} & \multicolumn{3}{|c|}{ Mujeres $(n=312)$} \\
\hline & SSPS & $\mathrm{N}$ & $\mathrm{P}$ & SSPS & $\mathrm{N}$ & $\mathrm{P}$ & SSPS & $\mathrm{N}$ & $\mathrm{P}$ \\
\hline BIDR & $-.42^{\star \star}$ & $-.35^{\star *}$ & $-.32^{\star *}$ & $-.41^{* *}$ & $-.32^{* *}$ & $-.31^{\star \star}$ & $-.42^{\star *}$ & $-.35^{\star \star}$ & $-.33^{\star *}$ \\
\hline $\mathrm{D}$ & $-.40^{\star \star}$ & $-.23^{* *}$ & $-.40^{\star *}$ & $-.37^{\star *}$ & $-.18^{\star *}$ & $-.39^{\star \star}$ & $-.41^{* *}$ & $-.27^{\star \star}$ & $-.39^{* *}$ \\
\hline I & $-.13^{\star \star}$ & $-.20^{\star \star}$ & $-.01 n s$ & $-.06 n s$ & $-.17^{\star \star}$ & $.07 n s$ & $-.19^{\star \star}$ & $-.23^{\star \star}$ & $-.09 n s$ \\
\hline AED & $-.43^{\star \star}$ & $-.26^{\star *}$ & $-.42^{\star \star}$ & $-.42^{\star \star}$ & $-.22^{\star *}$ & $-.44^{\star \star}$ & $-.44^{\star \star}$ & $-.32^{\star *}$ & $-.40^{\star *}$ \\
\hline $\mathrm{AEI}$ & $-.19^{\star \star}$ & $-.25^{\star *}$ & $-.06 n s$ & $-.14^{*}$ & $-.20^{\star *}$ & $-.02 n s$ & $-.25^{\star \star}$ & $-.30^{* *}$ & $-.11 n s$ \\
\hline MID & $-.23^{\star \star}$ & $-.12^{\star \star}$ & $-.24^{\star \star}$ & $-.20^{\star \star}$ & $-.08 n s$ & $-.23^{\star \star}$ & $-.23^{\star \star}$ & $-.13^{*}$ & $-.25^{\star *}$ \\
\hline MII & $-.04 n s$ & $-.11^{*}$ & $.04 n s$ & $.03 n s$ & $-.10 n s$ & $.13^{*}$ & $-.09 n s$ & $-.10 n s$ & $-.04 n s$ \\
\hline
\end{tabular}

${ }^{* *} p \leq .01,{ }^{*} p \leq .05, n s p>.05$. BIDR = Suma de los 40 ítems puntuados todos en sentido de deseabilidad social. D = Suma de los 20 ítems positivos o directos. I = Suma de los 20 ítems negativos con las puntuaciones invertidas. AED = Suma de los 10 ítems directos de Autoengaño. AEI = Suma de los 10 ítems negativos de Autoengaño con las puntuaciones invertidas. MID = Suma de los 10 ítems directos de Manejo de la Impresión. MII = Suma de los 10 ítems negativos de Manejo de la Impresión con las puntuaciones invertidas. SSPS = Suma de los 10 ítems puntuados todos en sentido de fobia social. SSPS-N = Suma de los 5 ítems directos o de autoverbalizaciones negativas (ítems 2, 4, 7, 8 y 10). SSPS-P = Suma de los 5 ítems negativos con las puntuaciones invertidas o autoverbalizaciones positivas (ítems 1, 3, 5, 6 y 9). 


\section{Diferencias de medias por sexo contro- lando la deseabilidad social}

Debido a la correlación significativa de la deseabilidad social con la escala SSPS, especialmente en mujeres, se estimaron de nuevo las diferencias por sexos controlando el puntaje total de BIDR por análisis de covarianza. Aplicado el análisis las medias entre hombres y mujeres resultaron equivalentes en el puntaje total y los dos factores de SSPS (véase tabla 6).

Tabla 6.

Modelo general lineal de SSPS, SSPS-N y SSPS-P introduciendo como covariante el puntaje total de BIRD y factor de efectos fijos el sexo

\begin{tabular}{|c|c|c|c|c|c|}
\hline$F V$ & $V D$ & $S C$ & $g l$ & $M C$ & $F$ \\
\hline \multirow{3}{*}{$\begin{array}{l}\text { Modelo } \\
\text { corregido }\end{array}$} & SSPS & $3618.92^{a}$ & 2 & 1809.46 & $67.30^{* *}$ \\
\hline & SSPS-N & $937.57^{b}$ & 2 & 468.78 & $42.42^{\star \star}$ \\
\hline & SSPS-P & $873.28^{c}$ & 2 & 436.64 & $36.46^{\star *}$ \\
\hline \multirow{3}{*}{ Intercepto } & SSPS & 13835.46 & 1 & 13835.46 & $514.58^{\star *}$ \\
\hline & SSPS-N & 3218.84 & 1 & 3218.84 & $291.26^{\star *}$ \\
\hline & SSPS-P & 940.45 & 1 & 940.45 & $78.54^{\star *}$ \\
\hline \multirow{3}{*}{ BIDR } & SSPS & 3449.65 & 1 & 3449.65 & $128.30^{* *}$ \\
\hline & SSPS-N & 885.07 & 1 & 885.07 & $80.09^{* *}$ \\
\hline & SSPS-P & 840.05 & 1 & 840.05 & $70.15^{\star \star}$ \\
\hline \multirow{3}{*}{ Sexo } & SSPS & 6.56 & 1 & 6.56 & $0.24 n s$ \\
\hline & SSPS-N & 3.78 & 1 & 3.78 & $0.34 n s$ \\
\hline & SSPS-P & .38 & 1 & 0.38 & $0.03 n s$ \\
\hline \multirow{3}{*}{ Error } & SSPS & 16696.89 & 621 & 26.89 & \\
\hline & SSPS-N & 6862.93 & 621 & 11.05 & \\
\hline & SSPS-P & 7436.31 & 621 & 11.97 & \\
\hline \multirow{3}{*}{ Total } & SSPS & 256151 & 624 & & \\
\hline & SSPS-N & 57395 & 624 & & \\
\hline & SSPS-P & 244962 & 624 & & \\
\hline \multirow{3}{*}{$\begin{array}{c}\text { Total } \\
\text { corregido }\end{array}$} & SSPS & 20315.81 & 623 & & \\
\hline & SSPS-N & 7800.50 & 623 & & \\
\hline & SSPS-P & 8309.59 & 623 & & \\
\hline
\end{tabular}

a. $R^{2}=.18$ y $R^{2}{ }_{a j .}=.17$, b. $R^{2}=.12$ y $R_{a j .}^{2}=.12$, c. $R^{2}=.10$ y $R_{a j .}^{2}=.10 .{ }^{* *} p \leq .01,{ }^{\star} p \leq .05$, $n s p>.05$.

SSPS = Suma de los 10 ítems puntuados todos en sentido de fobia social. SSPS-N = Suma de los 5 ítems directos o de autoverbalizaciones negativas (ítems 2, 4, 7, 8 y 10). SSPS-P = Suma de los 5 ítems negativos con las puntuaciones invertidas o autoverbalizaciones positivas (ítems $1,3,5,6$ y 9 ). 


\section{Discusión}

La curtosis multivariada de Mardia de los 10 ítems fue mayor que 10 y la razón crítica mayor que 3 , lo que refleja incumplimiento del supuesto de normalidad multivariada. No obstante, la curtosis multivariada de Mardia fue menor que 70 , lo que refleja que la desviación de la normalidad multivariada está en un rango tolerable para aplicar métodos de estimación de la función de discrepancia que la asumen (Rodríguez \& Ruiz, 2008). Así, en el análisis factorial confirmatorio, se optó por Mínimos Cuadrados Generalizados que se desarrollaron como un método alternativo a máxima verosimilitud. Aunque posee las mismas asunciones, como normalidad multivariada, la ponderación generalizada que este método introduce en la función de discrepancia puede corregir sesgos en las distribuciones, especialmente si son ligeras desviaciones de la normalidad (Cragg, 1983). Cuando la desviación es fuerte o el tamaño de muestra es pequeño el método de Mínimos Cuadrados Generalizados incurre en sesgos de estimación importantes, siendo preferible otro método, como el estimador asintóticamente libre de distribución (Muthén \& Kaplan, 1992).

En esta muestra de estudiantes mexicanos se validó la estructura hipotetizada de dos factores correlacionados de autoverbalizaciones positivas y autoverbalizaciones negativas en ambos sexos. Esta estructura también fue estable en Estados Unidos de América tanto en población de estudiantes como en población clínica de pacientes diagnosticados de fobia social (Hofmann \& DiBartolo, 2000). A su vez, este resultado coincide con los obtenidos en España con pacientes fóbicos (Gallego et al., 2010) y adolescentes de población general (Rivero et al., 2010).

Debe juzgarse el ajuste en el contraste unigrupo como bueno, ya que 5 de los 7 índices mostraron buen ajuste y uno adecuado. El único índice con mal ajuste fue el más sensible al incumplimiento del supuesto de normalidad multivariada y que se ve afectado por tamaños muestrales grandes $(N>400)$, el estadís- tico chi-cuadrado (Kline, 2010). También debe valorarse como buenas las propiedades de invarianza entre hombres y mujeres, ya que todos los parámetros fueron significativos en dos muestras en los cuatro modelos anidados, los valores de ajuste fueron buenos en términos generales, quitando peso al estadístico chicuadrado y tres modelos tuvieron una bondad de ajuste equivalente. El modelo con más constricciones y grados de libertad, al requerir una equivalencia exacta en residuos, mostró peor ajuste a los modelos con menos constricciones, pero éste fue adecuado en términos generales (Byrne, 2008).

Como se esperaba la consistencia interna fue alta para el conjunto de 10 ítems, siendo mayor la consistencia para las autoverbalizaciones negativas que para las autoverbalizaciones positivas. No obstante, los valores de consistencia interna de los dos factores fueron adecuados. En este estudio realizado en Méxi$\mathrm{co}$, la consistencia fue menor que la reportada en los estudios de Hofmann y DiBartolo (2000) en Estados Unidos de América, y de Gallego et al. (2010) y Rivero et al. (2010) en España.

Conforme a las expectativas las distribuciones del puntaje total y sus factores fueron asimétricas y no se ajustaron a una curva normal (Antony \& Rowa, 2008; García et al., 2003). Por lo tanto, la escala debería ser estandarizada por los percentiles. Las puntuaciones se concentraron en los valores bajos, lo que es característico de escalas que evalúan aspectos de conducta anormal o psicopatología. También este fenómeno se da en rasgos o conductas condenadas socialmente o vergonzosas y, en consecuencia, contaminadas por el sesgo de la deseabilidad social (Paulhus, 2002). En la presente muestra la escala SSPS sí correlacionó con la deseabilidad social y la varianza compartida fue del 18\%; por lo cual el efecto fue moderado y requeriría su control. El autoengaño tuvo más peso que el manejo de la impresión y se evidenció más en las autoverbalizaciones positivas y en las mujeres, aunque también es- 
tuvo presente en autoverbalizaciones negativas y en los hombres.

Debe señalarse que los promedios hallados en este estudio fueron más altos que en los reportados para las muestras estadounidenses de estudiantes del estudio original (Hofmann \& DiBartolo, 2000) y se aproximaron más a los parámetros de población general adolescente del estudio de Rivero et al. (2010) realizado en España. Esto probablemente se deba a la estimación más precisa del segundo estudio, ya que las muestras del primer estudio fueron incidentales y algunas incluso pequeñas.

Como se esperaba el promedio de los hombres fue más alto que el de las mujeres (Hofmann \& DiBartolo, 2000; Turk, et al., 2005). Los hombres se mostraron más propensos a las autoverbalizaciones negativas, pero al controlar el sesgo de la deseabilidad social esta diferencia desapareció. Así se interpreta que las mujeres reportan menos autoverbalizaciones negativas al afrontar situaciones estresantes de hablar en público que los hombres a causa de una menor crítica consigo mismas (factor de autoengaño de la deseabilidad social).

El promedio en las autoverbalizaciones negativas fue significativamente menor que el de las autoverbalizaciones positivas, como reportan estudios previos (Gallego et al., 2010; Hofmann \& DiBartolo, 2000; Hofmann, Moscovitch, Kim \& Taylor, 2004; Rivero et al., 2010). No obstante, al controlar el efecto del autoengaño (ítems directos), la diferencia desapareció $(p=.86)$, cuando el efecto de la interacción del autoengaño con la modalidad de autoverbalizaciones fue significativo $(p<.01)$. Nuevamente el sesgo de la deseabilidad social está presente en la escala, lo que evidencia la necesidad de controlarlo.

Debido a la composición diferencial de mujeres y hombres entre las dos facultades, el contraste multigrupo del modelo factorial se realizó también por facultades. Las propiedades de invarianza entre las dos facultades fueron buenas por la significación de parámetros, índices de ajuste y equivalencia de bondad de ajuste entre tres modelos anidados. Esto aunado a que la facultad no fue un factor diferencial en los promedios de la puntuación total y los factores de SSPS, esto es, no fue una covariante significativa, se puede afirmar que la invarianza factorial entre ambos sexos y sus diferencias en promedios poseen una atribución clara no contaminada por el hecho de que hubo más mujeres procedentes de Psicología y más hombres procedentes de Orientación Educativa.

Como limitaciones del estudio debe señalarse que, aunque se cuenta con una muestra probabilística estratificada por sexos, las estimaciones sólo serían válidas para los estudiantes de Psicología y Orientación Educativa de la universidad donde se hizo el estudio. En poblaciones afines, como en otras facultades de la misma universidad o en las mismas facultades de otras universidades, estos resultados deben manejarse como hipótesis. Aparte, son datos de autorreporte que pueden diferir de los obtenidos por métodos de entrevista, observación, experimentales o proyectivos.

En conclusión, se validó la estructura de dos factores correlacionados de autoverbalizaciones positivas y negativas, teniendo buenas propiedades de invarianza entre ambos sexos. La consistencia interna de la escala fue alta, aunque adecuada la de los factores. Las distribuciones fueron asimétricas, de ahí que la escala debe ser estandarizada por los percentiles. Se observó un efecto moderado de la deseabilidad social sobre la escala, especialmente del aspecto del autoengaño que requiere su control estadístico. Como en otros estudios se evidenció mayor promedio en hombres y autoverbalizaciones positivas, pero al controlar por análisis de covarianza el efecto del autoengaño estas diferencias desaparecieron.

Como se señaló en la introducción, es importante estudiar la fobia social específica en la situación de hablar en público en estudiantes universitarios y futuros profesionales, pero esta 
población tiene unas características peculiares que la diferencian de la población general. Así se recomienda el uso de esta escala en población de estudiantes universitarios mexicanos, y se sugiere replicar este estudio con otras muestras probabilísticas de población general y clínica, también con una porción equivalente de sexos, incluso dentro de cada submuestra, para contrastar los presentes resultados.

Es importante señalar que, de este estudio, se desprende la necesidad de considerar el efecto de la deseabilidad social en la investigación de la fobia social, que es un aspecto desatendido en los estudios publicados.
American Psychiatric Association (2000). Diagnostic and statistical manual of mental disorders, fourth edition, text revision (DSM-IV-TR). Washington, DC: American Psychiatric Association. http://dx.doi. org/10.1176/appi.books.9780890423349

American Psychological Association (2002). Ethical principles of psychologists and code of conduct. American Psychologist, 57, 1060-1073. http://dx.doi.org/10.1037/0003-066X.57.12.1060

Antona, C. (2009). Fobia social: evaluación y tratamiento. México, DF: Trillas.

Antona, C. J., Delgado, C., García, L. J. \& Estrada, B. (2012). Adaptación transcultural del tratamiento IAFS en estudiantes mejicanos con fobia social: un estudio piloto. International Journal of Psychology and Psychological Therapy, 14, 35-48.

Antona, C. \& García, L. J. (2008). Repercusión de la exposición y reestructuración cognitiva sobre la fobia social. Revista Latinoamericana de Psicología, 40, 281-292.

Antony, M. M. \& Rowa, K. (2008). Social anxiety disorder: Psychological approaches to assessment and treatment. Göttingen, Germany: Hogrefe and Huber.

Antony, M. M., Rowa, K., Liss, A., Swallow, S. R. \& Swinson, R. P. (2005). Social comparison processes in social phobia. Behavior Therapy, 36, 65-75. http://dx.doi.org/10.1016/S0005-7894(05)80055-3

Arbuckle, J. L. (2007). AMOS 16.0 user's guide. Spring House, PA: Amos Development.

Beck, A. T., Ward, C. H., Mendelson, M., Mock, J. \& Erbaugh, J. (1961). An inventory for measuring depression. Archives of General Psychiatry, 4, 561-571. http://dx.doi.org/10.1001/archpsyc.1961.01710120031004

Byrne, B. M. (2008). Testing for multigroup equivalente of a measuring instrument: A walk through the process. Psicothema, 20, 872-882.

Cragg, J. G. (1983). More efficient estimation in the presence of heteroscedasticity of unknown form. Econometrica, 51, 751-764. http://dx.doi.org/10.2307/1912156

Cronbach, L J. \& Shavelson, R. J. (2004). My current thoughts on coefficient alpha and successor procedures. Educational and Psychological Measurement 64, 391-418. http://dx.doi. org/10.1177/0013164404266386

Gallego, M. J., Botella, C., García, A., Quero, S., \& Baños, R. M. (2010). La escala de "Autoverbalizaciones durante la situación de hablar en público" (SSPS): propiedades psicométricas en una muestra española de fóbicos sociales. Psicología Conductual, 18, 343-363.

García, C. H. (2009). Cómo investigar en psicología. México: Trillas.

García, L. J., Olivares, J. \& Vera, P. E. (2003). Social anxiety disorder: Revision of assessment measures for Spanish-speaking population. Revista Latinoamericana de Psicología, 35, 151-160.

Glass, C. R., Merluzzi, T. V., Biever, J. L. \& Larsen, K. H. (1982). Cognitive assessment of social anxiety: Development and validation of a selfstatement questionnaire. Cognitive Therapy and Research, 6, 37-55. http://dx.doi.org/10.1007/BF01185725

Heinrichs, N. \& Hofmann, S. G. (2001) Information processing in social phobia: A critical review. Clinical Psychology Review, 21, 751-770. http://dx.doi.org/10.1016/S0272-7358(00)00067-2

Hofmann, S. G. (2007). Cognitive factors that maintain social anxiety disorder: A comprehensive model and its treatment implications. Cognitive Behaviour Therapy, 36(4), 195-209. http://dx.doi. org/10.1080/16506070701421313

Hofmann, S. G. \& DiBartolo, P. M. (2000). An instrument to assess self-statements during public speaking: Scale development and preliminary psychometric properties. Behavior Therapy, 31, 499-515. http://dx.doi.org/10.1016/S0005-7894(00)80027-1

Hofmann, S. G., Moscovitch, D. A., Kim, H. J. \& Taylor, A. N. (2004). Changes in self-perception during treatment of social phobia. Journal of Consulting and Clinical Psychology, 72, 588-596. http:// dx.doi.org/10.1037/0022-006X.72.4.588

Kline, R. B. (2010). Principles and practice of structural equation modeling (3rd ed.). New York: The Guilford Press.

MacCallum, R. C., Browne, M. W. \& Cai, L. (2006). Testing differences between nested covariance structure models: Power analysis and 
null hypotheses. Psychological Methods, 11, 19-35. http://dx.doi. org/10.1037/1082-989X.11.1.19

Moral, J. (2006). Análisis factorial confirmatorio. En R. Landero \& M. T. González (Eds.), Estadística con SPSS y metodología de la investigación (pp. 445-528). México, DF: Trillas.

Muthén, B. \& Kaplan, D. (1992). A comparison of some methodologies for the factor analysis of non-normal Likert variables: A note on the size of the model. British Journal of Mathematical and Statistical Psychology, 45, 19-30. http://dx.doi.org/10.1111/j.2044-8317.1992. tb00975.x

Paulhus, D. L. (1998). Manual for Balanced Inventory of Desirable Responding: version 7.(BIDR-7). Toronto, ON: Multi-Health Systems.

Pauhlus, D. L. (2002). Socially desirable responding: The evolution of a construct. En H. Brau, D. Jackson \& D. E. Wiley (Eds.), The role of constructs in psychological and educational measurement (pp. 4669). Mahwah, NJ: Lawrence Erlbaum.

Preacher, K. J. \& Coffman, D. L. (2006, Mayo). Computing power and minimum sample size for RMSEA [Computer software]. Disponible en: http://quantpsy.org/rmsea/rmsea.htm

Rivero, R., García, L. J. \& Hofmann, S. G. (2010). The Spanish version of the Self-Statements During Public Speaking scale: Validation in adolescents. European Journal of Psychology Assessment, 26(2), 129-135

Rodríguez, M. N. \& Ruíz, M. A. (2008). Atenuación de la asimetría y de la curtosis de las puntuaciones observadas mediante transformaciones de variables: Incidencia sobre la estructura factorial. Psicológica, 29(2), 205-227.

Sociedad Mexicana de Psicología (2007). Código ético del psicólogo. México, DF: Trillas.

SPSS Incorporation (2007). The SPSS base 16.0. User's guide. Chicago, IL: SPSS Inc.

Stewart, D. W. \& Mandrusiak, M. (2007). Social phobia in college students: A developmental perspective. Journal of College Student Psychotherapy, 22(2), 65-76. http://dx.doi.org/10.1300/ J035v22n02_06

Thrusfield, M., Ortega, C., de Blas, I. Noordhuizen, J. \& Frankena, K. (2001). Win Episcope 2.0. Improved epidemiological software for veterinary medicine. The Veterinary Record, 148(18), 567-572. http:// dx.doi.org/10.1136/vr.148.18.567

Turk, C. L., Heimberg, R. G., Luterek, J. A., Mennin, D. S. \& Fresco, D. M. (2005). Emotion dysregulation in generalized anxiety disorder: A comparison with social anxiety disorder. Cognitive Therapy and Research, 29, 89-106. http://dx.doi.org/10.1007/s10608-005-1651-1 


\section{Anexo. Escala de Autoverbalizaciones al Hablar en Público}

Por favor, recuerde lo que usted comúnmente ha sentido y pensado durante cualquier clase de situación en la que tuvo que hablar en público. Recordando estas situaciones, díganos qué tanto está de acuerdo con las oraciones de abajo. Por favor escoja el grado en que la frase representa lo que usted sintió o pensó en tales situaciones, en una escala de 1 (si está totalmente en desacuerdo) a 5 (si está totalmente de acuerdo).

\begin{tabular}{|c|c|c|c|c|}
\hline 1 & 2 & 3 & 4 & 5 \\
\hline $\begin{array}{c}\text { Totalmente en } \\
\text { desacuerdo }\end{array}$ & $\begin{array}{c}\text { Bastante en } \\
\text { desacuerdo }\end{array}$ & $\begin{array}{c}\text { Ni de acuerdo ni en } \\
\text { desacuerdo }\end{array}$ & Bastante de acuerdo & Totalmente de acuerdo \\
\hline
\end{tabular}

1. No tengo nada que perder, vale la pena el 1 intentarlo

2. Soy un perdedor

3. Ésta es una situación incómoda, pero puedo manejarla

4. Fracasar en esta situación sería una prueba más de mi incapacidad

5. Aún cuando las cosas salgan mal, no es una catástrofe

6. Puedo manejar cualquier cosa

7. Lo que yo diga probablemente sonará estúpido

8. Probablemente "la vaya a regar" de todas 1 maneras

9. En lugar de preocuparme, podría concentrarme 1 en lo que quiero decir

10. Me siento incómodo y tonto, se van a dar cuenta 1 de cómo me siento

$\begin{array}{llll}2 & 3 & 4 & 5 \\ 2 & 3 & 4 & 5 \\ 2 & 3 & 4 & 5 \\ 2 & 3 & 4 & 5 \\ 2 & 3 & 4 & 5 \\ 2 & 3 & 4 & 5 \\ 2 & 3 & 4 & 5 \\ 2 & 3 & 4 & 5 \\ 2 & 3 & 4 & 5 \\ 2 & 3 & 4 & 5\end{array}$

5

5

5

5

5

5

5

5

5 